\title{
Proposal for a universal minimum information convention for the reporting on the derivation of human embryonic stem cell lines
}

\author{
Emma L Stephenson ${ }^{1,2}$, \\ Peter R Braude ${ }^{2,3}$ \& \\ Chris Mason ${ }^{1 \dagger}$ \\ ${ }^{\dagger}$ Author for correspondence \\ ${ }^{1} A d v a n c e d$ Centre for \\ Biochemical Engineering, \\ University College L ondon, \\ London, UK \\ 'In this editorial, we highlight the \\ variation in data published and voice \\ suggestionsas to how the reporting can \\ become standardized and so facilitate \\ progress in human embryonic \\ stem cell derivation.'
}

Tel.: +1 2076790140

Fax: +1 2072090703

E-mail: chris.mason@ ucl.ac.uk

${ }^{2} \mathrm{D}$ epartment of Women's $\mathrm{H}$ ealth, School of M edicine, King's College L ondon, London, UK

${ }^{3}$ Assisted Conception Unit, Guys and St Thomas' Foundation Trust, London, UK

\section{Karen Rowland, Graphics Editor} Future M edicine, Unitec H ouse, 2 Albert Place, Finchley Central, London, N 3 1Q B, UK

Tel.: +44 (0)2083492033

Fax: +44 (0)20 83432313

E-mail: k.rowland@

futuremedicine.com

The implementation of novel methodology across the human embryonic stem cell (hESC) field is fraught with difficulties, not least of which is the highly variable reporting of the number and quality of embryos used to derive each new hESC line. It is almost impossible to ascertain from the literature a true efficiency for deriving a hESC line. Until there is a clear minimum information (MI) convention amongst the derivation teams, understanding and comparing novel derivation methodologies and their potential impact on the resulting stem cell extremely difficult. In this editorial, we highlight the variation in data published and voice suggestions as to how the reporting can become standardized and so facilitate progress in human embryonic stem cell derivation. The authors request that interested parties comment line will continue to be impossible or at best on the system proposed, in order that a consensus article can be published in approximately 4 months time.

There is no universally agreed method for reporting the quality and type of embryo used as starting material for hESC line derivation. Whilst many groups have published their hESC derivation data and protocols, few have stated in their manuscripts the complete essential underlying metadata with respect to their source material to enable reliable comparisons to be made. However, it is beginning to be acknowledged that this lack of an accepted and established reporting system results in several hurdles for stem cell researchers. In clinicalassisted reproduction, standards for embryo and blastocyst quality have been devised that correlate with likelihood of implantation, and might beneficially be applied to stem cell derivation.

$M$ any advanced fields of biology have benefited greatly from the creation of their own bespoke reporting standards. These evolving standards have enabled all the researchers in a particular field to make the most of one another's data. Successful examples include the Standard Metabolic Reporting Structures Working Group [1], European Centre for the Validation of Alternative M ethods (ECVAM) Task Force [2] and genomic sequencing communities, such as Flybase, Saccharomyces Genome Database and the Mouse Genome $D$ atabase [3]. The benefits to the respective communities of standardization in reporting methodologies and the related metadata include "assisting innovation by sharing best practice and setting benchmarks for quality, safety and performance" [101]. Simplified annotation of the metadata also al lows for less cluttering of results and text, while continuing to relay a complete set of all the relevant underlying information. Thus, a standardized metadata format has a number of clear advantages in that "it facilitates comparisons, reposition and exchange of data" [4], which in turn has al ready resulted in a number of life science sectors extracting the maximum value from their collective data [5].

Development of a biological standard Two main approaches can be adopted in order to develop standards: a 'top-down' approach, where an umbrella organization mandates a standard, or a 'bottom-up' approach, where an evolving set of standards is produced by the end-users themselves. Whilst each has their merits and can be successfully applied, a bottom-up approach is more user friendly, has greater voluntary buy-in and suits fields that are rapidly evolving [3].

There is little debate over the value of the development of standards for biological areas of research, including the hESC field [6,7]. Standards in biology are not new. Since the 1800s, biological standards have been in place with one of the first recorded being the 1820 US Pharmacopoeia Standards for plant extracts used as medication [102]. There are many examples of more recent reports on the successful deployment of biological standards $[3,4,8]$. 
U niversally agreed key steps include:

- Identify the scope of the information that needs to be captured in the standard;

- Develop a simple and easy-to-read framework for the information that is needed to be included in order to develop a minimum information checklist or specification;

- $M$ ake the draft minimum information specification freely available;

- Develop a consensus view of the minimum information that needs to be reported;

- Circulate widely the agreed protocol in order that the metadata can be correctly incorporated into reports, publications, grant applications and the general dissemination of data;

- Standards are an ongoing process, therefore care must be taken to ensure that they are developed as the field progresses and new technologies evolve $[3,4,8]$.

\section{Minimum information}

The idea of a M I checklist or specification is a well tried and tested approach with a host of successful biological examples available from the fields of in situ hybridization and immunohistochemistry [9], flow cytometry [10] and phylogenetic analysis [11].

'The a im is that this editorial begins the 'bottom-up' debate to identify the need and scope of the minimum information metadata to be captured in the future standard for derivation of hESC lines.'

The M I checklist needs to be a public source document providing the guidelines for researchers when reporting their work, journal editors and reviewers, funding bodies, scientific bodies, technology suppliers and regulatory bodies and licensing authorities, such as, for example in the UK, the Human Fertilisation and Embryology Authority (H FEA) [4].

\section{Community acceptance}

In order for any standard to have value, it must be both relevant and acceptable with wide or preferably universal adoption. Therefore, there is an underlying requirement to patiently manage the consensus building process. Traditionally, getting a biology-focused research community to buy-in has been a long interactive process. However, the hESC derivation community has demonstrated a great willingness to collaborate; a good example being the International Stem Cell Initiative (ISCI) [12]. Furthermore, there are no competing existing standards nor any that would require careful negotiations in order to be merged into one.

Absolute acceptance is only likely to be achieved by a combination of:

- Grass roots developmental effort;

- Community-wide adoption as a result of a genuine benefit to all stakeholders;

- Formal support by journal editors, regulatory agencies and funding bodies [4].

If the above combination can be achieved, high standards of reporting can be accomplished in the shortest timespan and benefit the entire stem cell community, both hESC line derivation teams and hESC researchers [4].

\section{Current reporting practices}

The data in Table 1 have been collated from the majority of the derivation papers in the literature. The data extracted relates to the number of embryos used, culture media, gas phases and the information provided about embryo quality. Although there are recent reports of novel derivation methods, such as from morula-stage embryos [13,14], late arrested embryos [14] and single blastomeres [15], these experimental methods have yet to be implemented widely. The table refers to more conventional derivation attempts using immunosurgery, mechanical isolation or whole plating. Several points merit discussion since they admirably illustrate the potential for future best practice, although they should not be read as criticism but as commentary based on the wisdom of hindsight.

Lanzendorf and colleagues gave blastocysts a grade of 1 to 3 depending on the state of expansion and the appearance of the inner cell mass (ICM) [16]. Although fully explaining their grading system, they only reported grades for blastocysts that were successful in giving rise to a stem cell line. It would have been helpful to know the grade of all blastocysts used, as it could be that an equal number with the same grade did not give rise to a line, or alternatively that there was a clear distinction between grades resulting in success or failure. $\mathrm{H}$ eins and collaegues discuss in detail their use of immunosurgery and whole plating [17]. Although they show some clear pictures, they also only reported the grade for blastocysts used successfully to generate their six lines. There is also no information 
regarding the total number of embryos or blastocysts used. Readers are thus unable to discern which method was more efficient, not necessarily from this paper in isolation as the numbers used were perhaps insufficient, but in conjunction with results from other groups investigating the same comparison.

By contrast, Pickering and colleagues discussed the morphology of the blastocysts used in great detail, importantly reporting data for both the successful and unsuccessful blastocysts [18]. In addition, they published pictures describing the wide variation in size and appearance of the ICM in blastocysts with apparent excellent morphology. The point is made that this may have important implications for stem cell derivation. It also highlights the observation that, although the criteria for derivation are similar to those for assisted reproduction, for hESC derivation, the focus should be on describing the ICM, with comments as necessary for features that distinguish the overall morphology and health of the blastocyst. Chen and coworkers presented a comprehensive table of data describing all the blastocysts used, including their quality and outcome [19]. Such results are constructive for other researchers attempting derivation with poor-quality embryos, and confirmed that the morphological score on day 3 is not reliable in predicting development to blastocyst. Similarly, M italipova and colleagues presented a table with morphological data for each blastocyst used, as well as the day used and outcome [20].

'No two hESC lines are identical, and
knowing the quality of the starting
material may help elucidate the
underlying differences.'

The citing of percentage efficiency can be misleading. As shown in Tadel, some reports are per embryo, some per blastocyst and some per blastocyst used. For example, Amit and ItskovitzEldor state a $43 \%$ derivation efficiency using their results of three lines out of five blastocysts [21], plus Thomson and colleagues reported three lines from 14 blastocysts, and Reubinoff and coworkers two lines from four blastocysts. From Tadel, it is apparent that Thomson had access to 20 blastocysts, although only 14 were regarded as suitable for use and formed the denominator. Reported efficiencies drop dramatically when the number of available embryos is taken into account. In the report by Findikli and colleagues, the derivation efficiency is quoted for immunosurgery as $26.6 \%$, with four lines derived, when in fact two of the stem cell colonies differentiated in very early passage and hence were not maintained in culture [22]. It is arguable as to exactly what point a hESC line should be deemed established; most would only consider a line established when it has been characterized and banked, although some early successful lines have been lost to infection. The report by Inzunza and colleagues raises this important question [23]. In their manuscript, they report the derivation of two permanent, fully characterized hESC lines and ten early lines not yet firmly established or characterized. To date, there is no consensus as to what exactly constitutes the definition of a hESC line. Some authors prefer to state a certain passage number, whilst others use the first stock of cryopreserved cells that can reliably be expanded to high passage. Consistent reporting would be beneficial for data analysis. However, it should be stressed that it is important to report these putative colonies, as they add valuable information to be extracted from embryo number and quality data. We consider that efficiency of derivation should be quoted for the number of embryos taken for this purpose ('intention to derive'), as this is the true quantity of starting material for which units have to consent patients and receive embryos in order to obtain the required number of lines.

Even when the grade of blastocysts is reported, the grading systems in use are not uniform. $\mathrm{H}$ ence, although tentative extrapolation of results is possible by studying the systems used, it would be more accurate, consistent and reliable to produce one amenable to all and easy to implement, in order to establish the range in quality of blastocysts that give the optimum efficiency for derivation. Groups could then compare their performance using the same quality of blastocysts in a consistent manner. No two hESC lines are identical, and knowing the quality of the starting material may help elucidate the underlying differences.

\section{Existing clinical grading systems}

Since a 'good' blastocyst is the preferred starting point required for successful stem cell derivation, and has the highest implantation potential when used to establish pregnancy after in vitro fertilization (IVF), it would seem appropriate to examine the systems used clinically for grading blastocysts and embryos. One of the most widely used is that of Gardner and colleagues, 

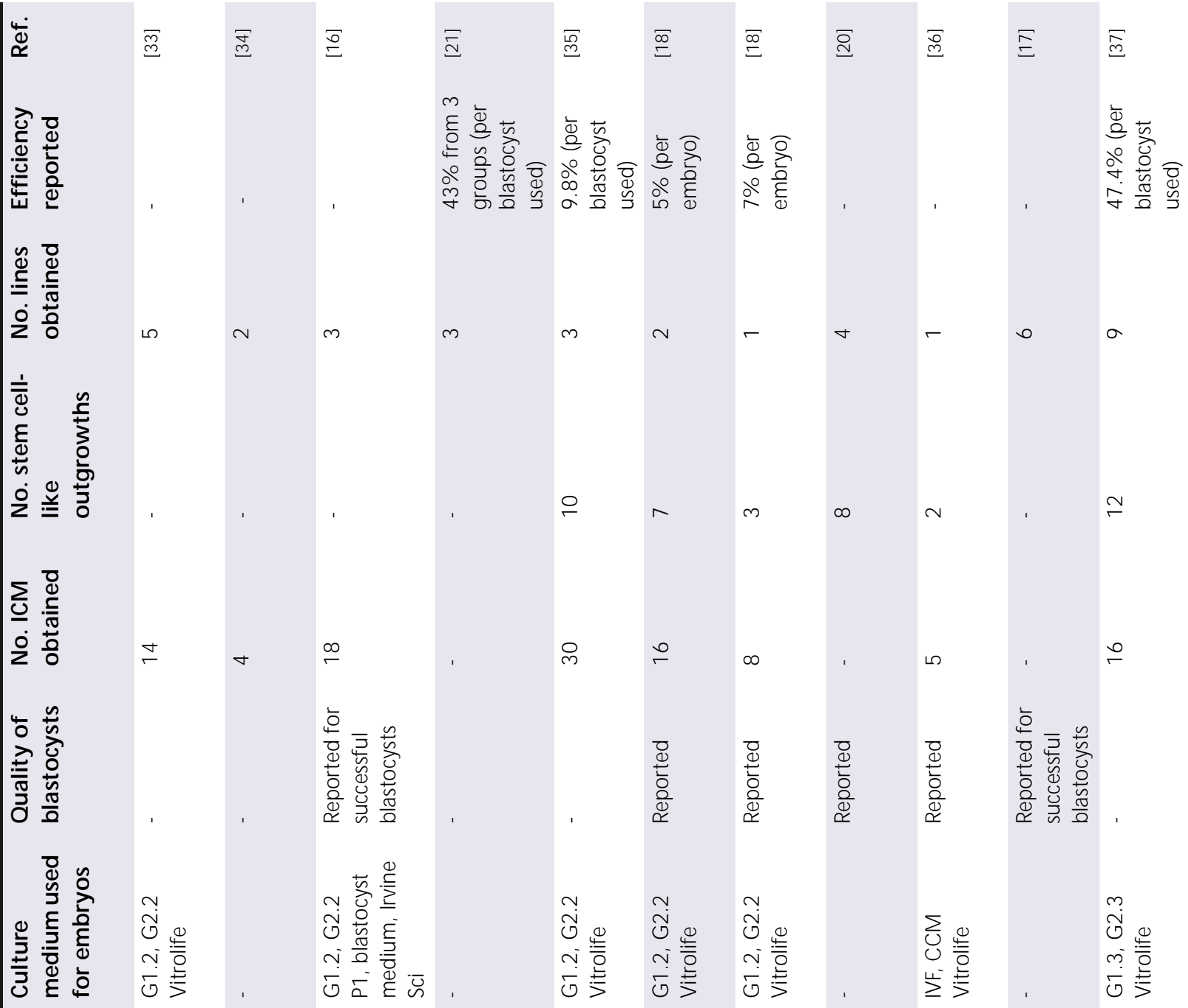

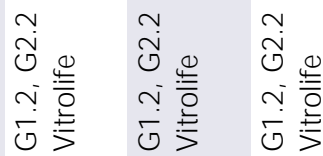

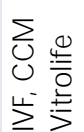

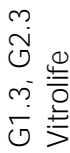
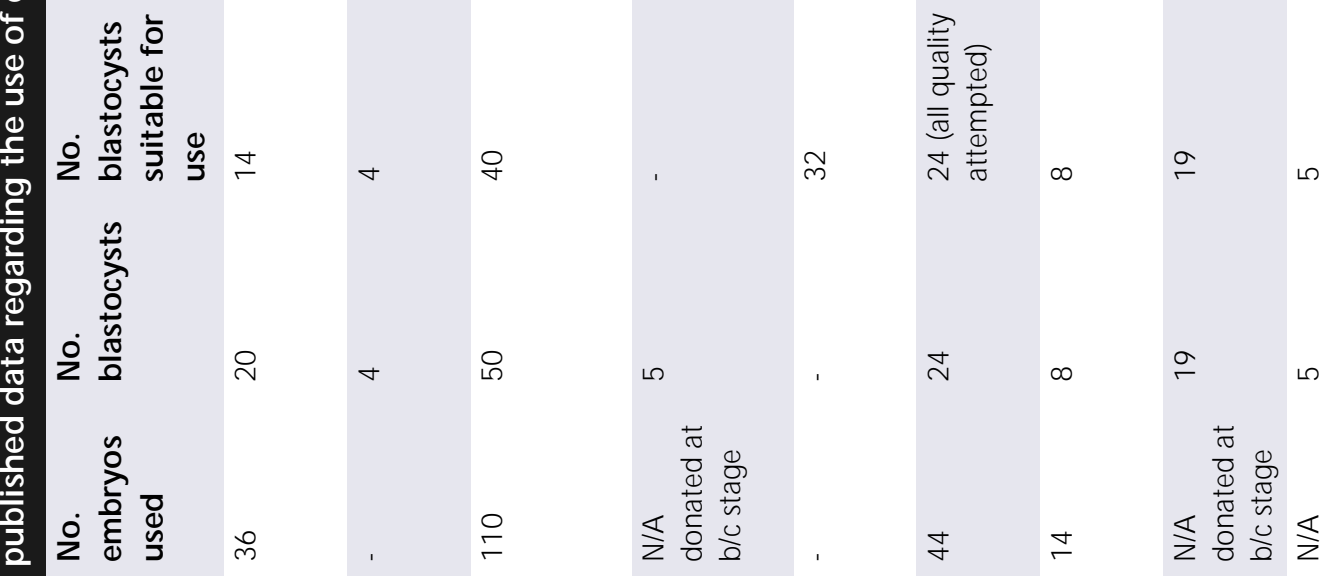

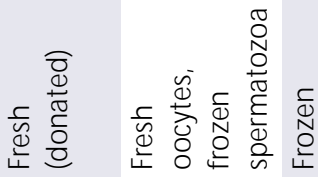

$\begin{array}{ll} & \widehat{0} \\ \frac{0}{0} & \frac{0}{2} \\ \stackrel{\frac{1}{4}}{\frac{0}{4}} & \frac{1}{4}\end{array}$

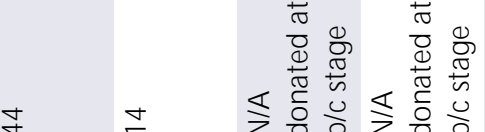

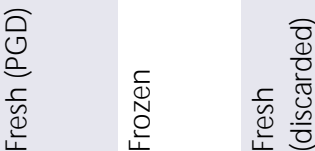

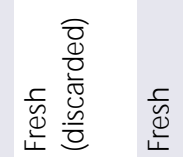




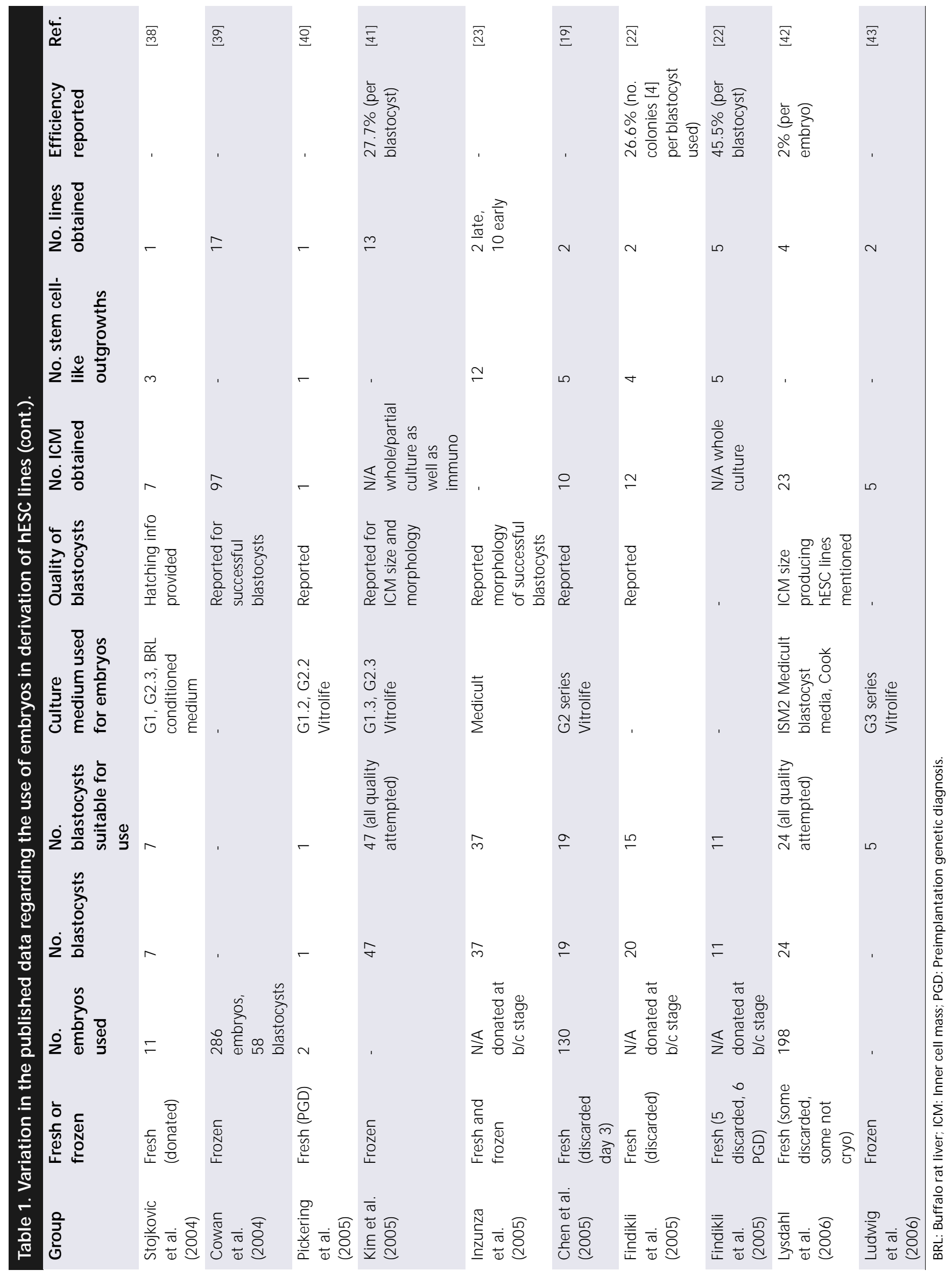


which was proposed in the 1990s [24]. In this system, the degree of blastocyst expansion is given a score of 1-6, and graded A-C for trophectoderm (TE) quality and ICM morphology, provided the blastocyst has reached expansion grade 3 or further. This system was modified by Veeck and Zaninovic in 2000, who extended the description of the TE and ICM to four categories, A-D [25]. While these systems were used successfully by the authors, who both reported positive correlation of high-grade blastocysts with good pregnancy rates, their systems were criticized in 2001 as being vague and poorly defined. Richter and colleagues analyzed 164 blastocysts, with respect to several parameters, in order to establish the optimum ICM size and shape for successfully establishing a pregnancy [26]. They stated that the system by Gardner and Veeck simply reflected differences in developmental timing rather than real differences in quality. They found quantitative measurements of the ICM were highly indicative, but not significant, of blastocyst implantation potential, with ICM s of a slightly oval shape (roundness index 1.04-1.20) most successful. Blastocyst diameter and TE cell numbers were not related to implantation rates in this study. This type of systematic study on blastocysts used in stem cell derivation could be helpful in defining optimum morphology. Regardless of the system chosen for use by a clinic, the recent publication by Arce and colleagues is reassuring [27]. They reported that embryo quality can be determined with a good degree of interobserver agreement, independent of the method of evaluation. In addition, the many embryologists involved in this multicenter trial classified embryos with excellent intraobserver reproducibility. H ence, if a standardized grading system is established for hESC derivation, researchers can be confident that the grading will be consistent and reproducible.

\section{Proposed MI standard for grading of blastocysts used in hESC derivation} All the current grading systems used to select the embryo(s) for transfer have been based on clinical IVF practice and experience. H owever, criteria for stem cell derivation are different; certainly the implantation rate of good quality blastocysts is much higher than the hESC derivation efficiency with similar grade embryos. It must be recognized that the genotype of the embryo as well as the morphology of the ICM are both significant factors. H owever, without the reporting of metadata, the understanding of these interactions may remain elusive. Although the quality of the TE is important as an overall indicator of embryo health since it establishes support for the embryo and ultimate formation of placenta, this may not be relevant in hESC derivation; indeed, TE is usually discarded or avoided. H owever, abnormalities in the TE may simply be easier to see, and as the development of the TE is intimately related to that of the ICM , may indicate developmental abnormalities in the ICM also. During whole-embryo plating there is perhaps a greater opportunity for intercellular interactions and ICM growth that could increase the likelihood of hESC derivation from an embryo with an initially poor ICM . H owever, many researchers report inhibition of derivation following whole plating due to TE overgrowth [18]. The appearance of the ICM may be a more important determining factor for successful derivation as opposed to conception, however it remains important to grade the TE, although not necessarily in the same detail as the ICM .

'If a standardized grading system is established for hESC derivation, researchers can be confident that the grading will be consistent and reproduc ible.'

With this in mind, Figre 1 shows a diagrammatic representation of our suggested blastocyst grading system for hESC derivation, adapted from that of Veeck and Zaninovic [25], and Figre2 shows photographic examples of the six grades of ICM presented in Figurel The grading system focuses on the development of the ICM and expansion status, with more general descriptions for the TE. W ith the thought that the ICM may be the dominant predictor, it could be that grades are reported in ranges more strongly based on ICM morphology. H ence, all blastocysts with a grade A ICM conceivably fall in the same category regardless of expansion or TE appearance. It is predictable, however, that many blastocysts will not fall neatly into one of the categories, as any system can only be a simplification of the continuous spectrum of developmental variance. Volume, cell number, density, shape, size, etc., may be the correlating factors - confirmation of what more accurately predicts derivation outcome requires systematic and accurate measurements of ICM features for a significant number of blastocysts. O nce these 
data are available, it will allow modification of the standard as part of the essential ongoing fine-tuning and validation.

Box 1 presents those factors that we consider essential to report for standardization, and others that we regard as desirable. It is hoped that reporting of this data becomes common practice. It is worth noting that collecting this data should not require additional work; almost certainly it is present in any laboratory's records, the only action required is to integrate the information into manuscripts for publication.

Reporting the number of embryos reaching each stage (i.e., donated; blastocyst; use; ICM ; attachment; outgrowth; lines) allows comparisons between protocols, enables feedback to the IVF clinics on culture systems and permits a more accurate cal culation regarding the efficiency of derivation. The use of an agreed grading system and inclusion of the grade of embryos, information about development, such as the day in culture they were ready to be used for derivation, and hatching status, enables an efficiency to be established for each range of grade. This will guide research groups and allow them to monitor progress or changes in protocol against a known expected outcome. Complete knowledge of the total number of embryos used to create a line provides real information concerning the likely number of embryos that will be required to fulfil the need once good manufacturing practice (GMP)-grade lines are being derived. If the embryos are fresh, it is also necessary to mention whether they were deemed of too poor quality to use for the patient and at what stage these were passed to research. D evelopment to the blastocyst in a significant number of these 'poor quality' embryos should require re-eval uation of the unit's criteria for selecting embryos in the patient's best interest. Where embryos are obtained from preimplantation genetic diagnosis (PGD) cycles, they are more likely to be as good qual ity as from fertile couples or created from donated gametes [18], but not suitable for transfer or clinical use as stem cells, since they are affected by a serious genetic condition. This data would permit enhanced interpretation of publications. The vast majority of centers culture embryos and hESCs in 5\% $\mathrm{CO}_{2}$ at $21 \% \quad \mathrm{O}_{2}$. As the field moves towards accepting the importance of culturing at physiological oxygen (1-5\%) and implements this gas phase, it will be essential to record this in derivation attempts and outcome. Indeed, it has recently been reported that derivation of mouse ESC s is more efficient at $5 \% \mathrm{O}_{2}$ [28]. By reporting the media used and the percentage and quality of blastocysts obtained, information can be gathered regarding the effectiveness of these commercially available proprietary media.

\section{Publication bias}

Publication bias is a recognized problem for consistency of reporting [29]. Groups that are unsuccessful in deriving a stem cell line tend not to report such data, and derivation by standard methods is commonly not sufficiently original to be accepted for publication. Fong and colleagues, who recently reported the unsuccessful derivation of a hESC line using pairs of blastomeres from 8-cell stage embryos, discuss the importance of publishing negative results such that other groups investigating similar approaches can learn from their experience and avoid going through the same discovery process, particularly when human embryos are a scarce and precious commodity [30].

In the UK at least, this problem has been addressed through regulation. In accordance with the licences granted by the HFEA for research using human embryos, every research group must report the number of embryos taken for stem cell research each year, and any stem cells derived must be lodged with the UK Stem Cell Bank [103]. Collating this information and making it available to all research groups would provide valuable data with which to improve technique and to estimate efficiency and future requirements. This process has been started as part of the activities of the ISCI and the UKbased $\mathrm{H}$ uman Embryonic Stem Cell C o-ordinators Network (hESCCO), an MRC-funded group that meet twice yearly, and amongst other roles strive to collate national information about embryo donation for stem cell derivation and to standardize the consent process for patients donating to research [104]. H owever, information on the quality of embryos used is still lacking and, since this is UK data only, an international effort is needed.

\section{Concluding remarks}

$O$ pen and transparent reporting of results will move forward the hESC field more rapidly towards clinical translation and application. The intention of this editorial has been to highlight this need and to suggest a framework in which data should be recorded. In this context, examples have been described that have benefited from such an approach, the current discrepancies in reporting have been discussed and 
EDITORIAL - Stephenson, Braude \& Mason

\section{Expansion status}

1
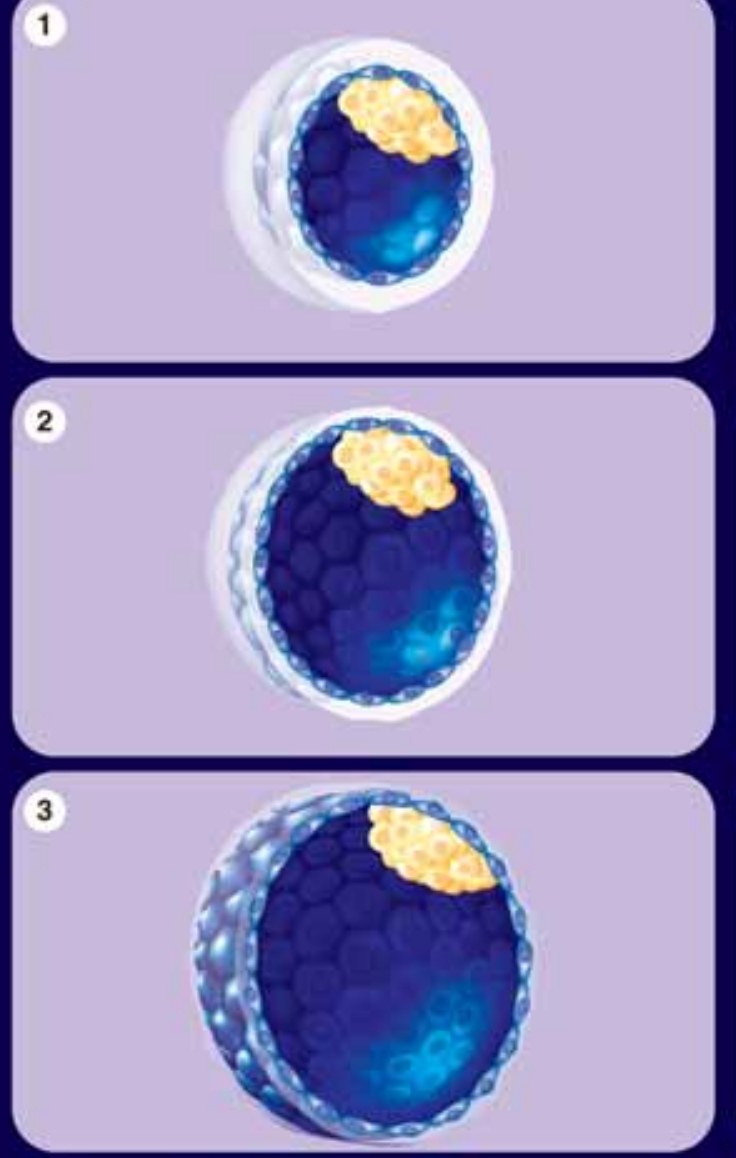

4
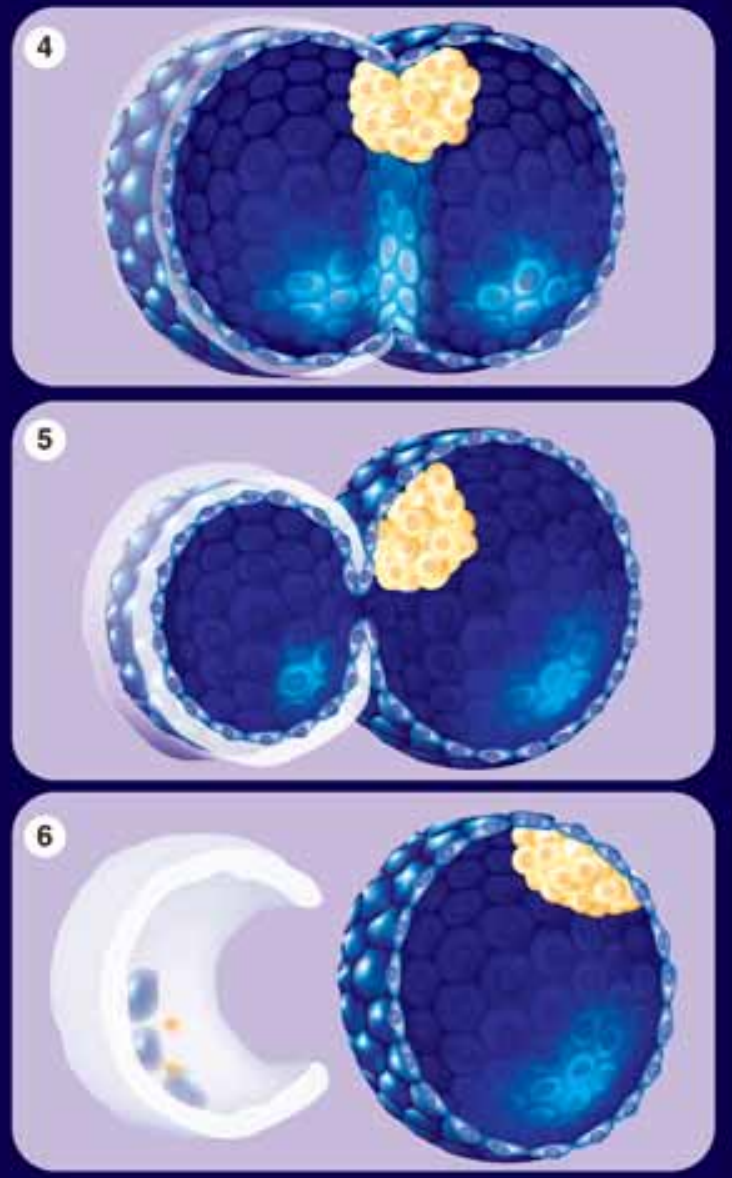

Inner cell mass appearance

A
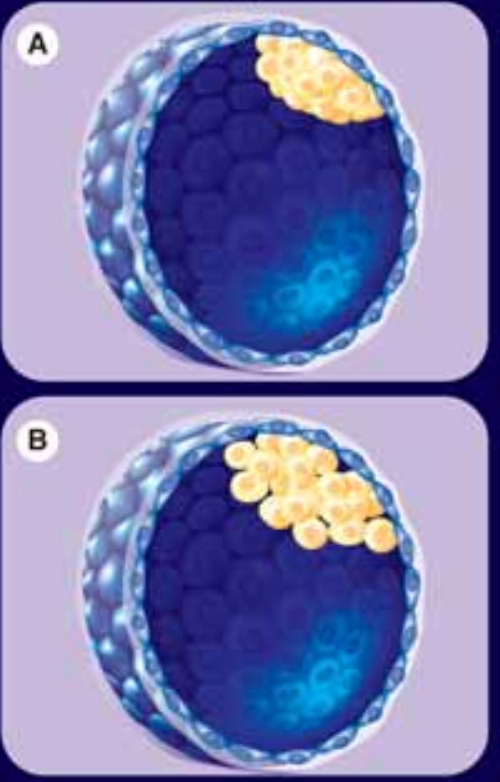

C

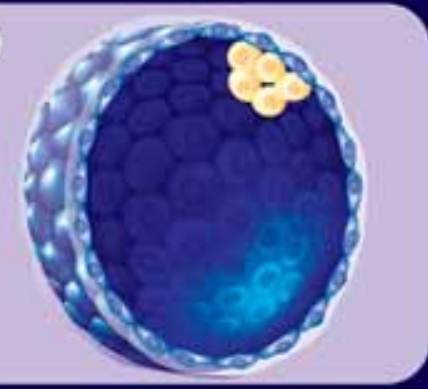

D

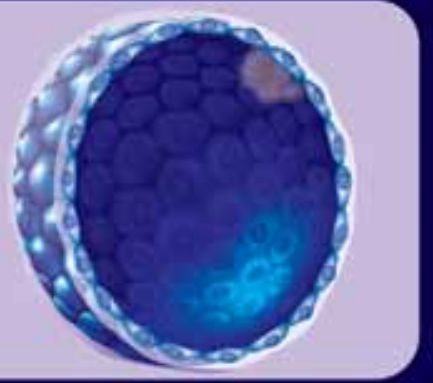

E

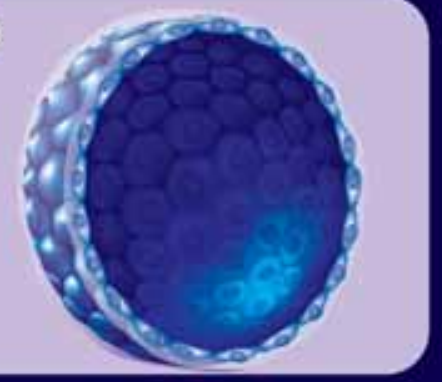

Key

Zona pellucida

Trophectoderm

Inner cell mass
Trophectoderm appearance
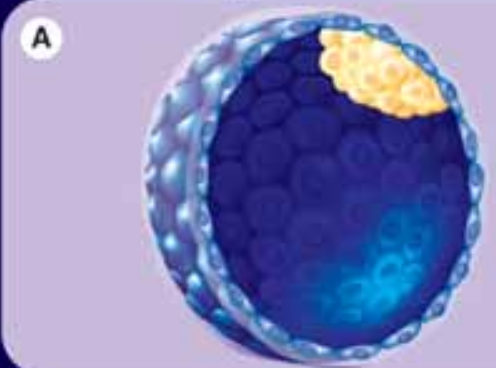

B

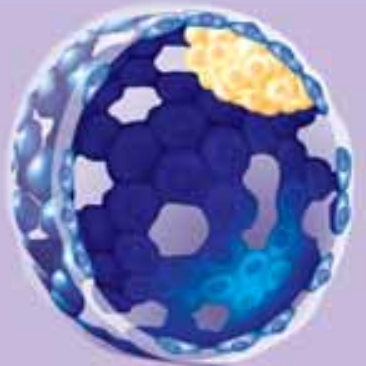

C

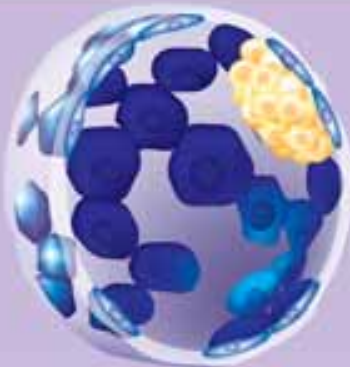

Expansion status

1 No expansion in overall size, zona pellucida still thick

2 Some expansion in overall size, zona pellucida beginning to thin

3 Full expansion, zona pellucida very thin

4 Partially hatched, trophectoderm beginning to herniate through zona pellucida. Full

expansion status

5 Partially hatched, no expansion in overall size seen following pre-implantation

genetic diagnosis

6 Fully hatched blastocyst, zona pellucida may contain cells rejected during blastocyst formation ICM appearance

(see figure 2, right, for photographic examples)

A Cells compacted, tightly adhered together and

indistinguishable as individual cells

B Cells less compacted so larger in size, loosely

adhered together, some visible as individual cells

C Very few cells visible, either compacted or loose,

may be difficult to completely distinguish from trophectoderm

D Cells of ICM appear degenerate

E No ICM cells visible in any plane

Trophectoderm appearance

A Many small identical cells forming a continuous trophectoderm layer

B Fewer, larger cells, may not form completely continuous layer

C Sparse cells, may be very large, very flat or appear degenerate 
Proposal for a convention for hESC derivation - EDITORLAL
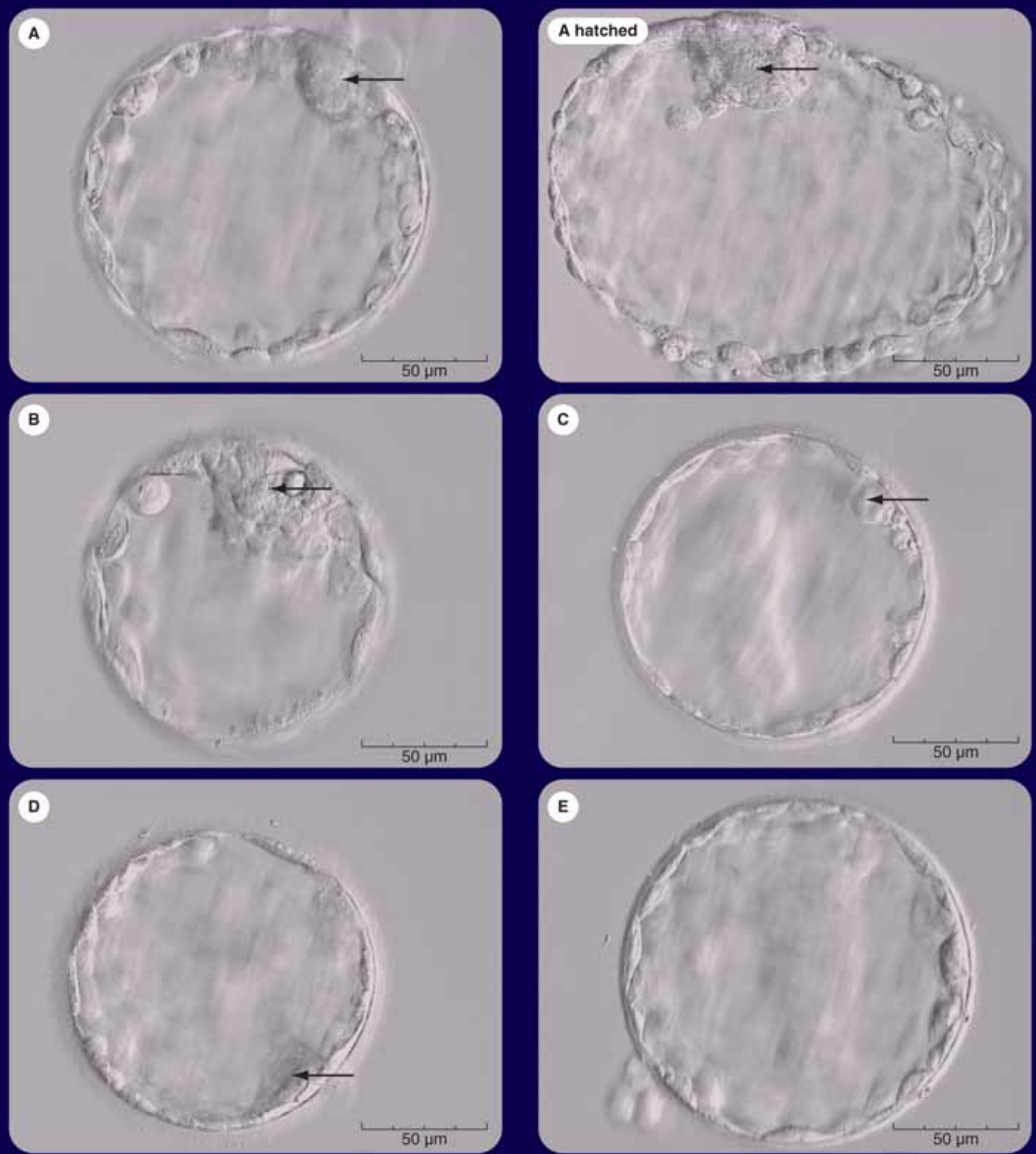

Figure 2 (above). Photographic examples of ICM grades A-E.

Photographic examples of the ICM appearance represented in diagrammatic form in Figure 1. These pictures demonstrate ICM grade A-E only, and do not consider expansion or trophectoderm appearance. It is important to scan through every plane of the blastocyst to confer an accurate grade. ICM indicated by arrow.

\section{Figure 1 (left). Proposed classification system for hESC derivation.}

Diagrammatic representation of the grading system proposed for blastocysts used in hESC derivation. Column 1 shows expansion status only; the ICM and trophectoderm are standard to highlight the variable. Similarly, Column 2 shows ICM appearance only, and Column 3 trophectoderm morphology only. 
a grading system has been proposed. In addition, the authors suggest a comprehensive guide to the data considered necessary to enable maximum information to be interpreted, to facilitate translation of protocols and establishment of the number of embryos required to fulfill clinical need.

This proposal of a M I convention for reporting on the derivation of hESC lines has already started to be discussed both with leading individuals in the field as well as interested organizations. H owever, this is just the beginning of the feedback process. In addition to being published in this journal, reprints of the article have been sent out to a broad collection of interested parties requesting feedback and suggestions.

'Interested parties a re encouraged to forward their views and comments as soon as possible in order that a consensus document can be published in the not too distant future.'

Box 1. Minimum information (MI) data deemed essential or desirable in publications using human embryos for stem cell research.

\section{Essential}

- Total number of embryos transferred to research

- Stage of embryos used

- Number developing to blastocyst

- Number of blastocysts suitable for use

- Day of development when blastocysts used

- Grade of all blastocysts used

- Number of ICM s obtained (if applicable) and method used to isolate

- Number of ICMs that attach to feeder layer/ECM (extracellular matrix)

- Number of days after plating until appearance of stem cell-like outgrowth

- Number of stem cell-like colonies

- Number of hESC lines

- Known disease (if PGD)

- Media used

- Gas phase employed $\left(\mathrm{CO}_{2}, \mathrm{O}_{2}\right)$

\section{Desirable}

- Source if fresh:

- Donated gametes

- Embryos deemed of too poor quality to cryopreserve

- No wish for cryopreservation

- PGD or pre-implantation genetic screening (PGS)

- Hatched naturally or zona pellucida removed (non-PGD)

- Passage number of the lines being reported

- Sex (useful for tracking cells)

hESC: Human embryonic stem cell; ICM : Inner cell mass;

PGD: Preimplantation genetic diagnosis.
The aim is that this editorial begins the "bottom-up' debate to identify the need and scope of the MI metadata to be captured in the future standard for derivation of hESC lines. We plan to adhere to the above guidelines for developing biological standards not only by making the document freely available (including free web access), but in particular actively seeking community input. The authors also acknowledge that if a standard is to have genuine long-term value it will require ongoing development. For the regenerative medicine industry to mature, agreed international standards are essential as they have been for every technology-based industry [31]. National standards are just starting to get underway for stem cells and regenerative medicine products [32].

Interested parties are encouraged to forward their views and comments as soon as possible in order that a consensus document can be published in the not too distant future. We hope that this will facilitate hESC research and translation and complement the admirable work of leading international organizations, such as the International Society for Stem C ell Research, the EU 6th Framework Programme funded collaboration EST O O LS and the UK Stem Cell Bank.

\section{Acknowledgements}

The authors would like to thank the following international experts from the hESC embryology/derivation community for their enthusiastic support, valuable insight and suggestions. Paul D e Sousa, Centre for Regenerative M edicine, College of M edicine \& Veterinary M edicine, University of Edinburgh, UK and Rosin Cells Ltd, Roslin Institute, Rosin, Midlothian, U K; D avid Gardner, Colorado Center for Reproductive Medicine, Englewood, CO, USA; O uti Hovatta, REGEA, Insitute for Regenerative M edicine, University of Tampere and Tampere University Hospital, Finland and Division of Obstetrics and Gynecology, Department of CLINTEC, Karolinska Institutet, Karolinska University Hospital Huddinge Stockholm, Sweden; Jennifer Nichols, Wellcome Trust Centre for Stem Cell Research, U niversity of Cambridge, Tennis Court Road, Cambridge, UK; Susan Pickering Edinburgh Fertility and Reproductive Endocrine Centre, Assisted Conception Programme, Simpson Centre for Reproductive Health, Royal Infirmary of Edinburgh, Edinburgh, UK; Glyn Stacey, UK Stem Cell Bank and D ivision of $\mathrm{C}$ ell Biology and Imaging, $\mathrm{N}$ ational Institute for Biological Standards and Control (NIBSC), South M imms, Potters Bar, UK; M iodrag Stojkovic, Sintocell, Leskovac, Serbia; Alan Trounson, M onash Immunology and Stem Cell Laboratories, M onash U niversity, Melbourne, Victoria, Australia; Anna Veiga, Banc de Linies Cellulars Centre de M edicina Regenerativa de Barcelona, Barcelona. E.L.S. is supported by the M RC, BBSRC and Guysand St Thomas' Charity. 
Bibliography

1. Lindon JC, N icholson JK, $\mathrm{H}$ olmes $\mathrm{E}$ et al.: Summary recommendations for standardization and reporting of metabolic analyses. $\mathrm{N}$ at. Biotechnol. 23, 833-838 (2005).

2. Coecke $S$ et al.: Good cell culture practice guidance. Altern. Lab. Anim. 33, 261-283 (2005).

3. Brooksbank C, Q uackenbush J: D ata standards: a call to action. O M ICS 10 , 94-99 (2006).

4. Field D, Sansone SA: A special issue on data standards. O M ICS 10, 84-93 (2006).

5. Q uackenbush J: D ata standards for 'omic' science. $\mathrm{N}$ at. Biotechnol. 22, 613-614 (2004).

6. Brivanlou AH, Gage FG, Jaenisch R, Jessell T, M elton D, Rossant J: Setting standards for human embryonic stem cells. Science 300, 913-916 (2003).

7. Veiga A, Camarasa M V, Aran B, Raya A, Izpisua JC: Stem cells in human reproduction basics and therapeutic potential. Informa H ealthcare. (2006) (In Press).

8. Brazma A, H ingamp P, Q uackenbush J et al.: M inimum information about a microarray experiment (M IAM E)-toward standards for microarray data. $N$ at. $G$ enet. 29, 365-371 (2001).

9. D eutsch EW, Ball CA, Bova GS et al.: $D$ evelopment of the $M$ inimum Information Specification for In Situ $\mathrm{H}$ ybridization and Immunohistochemistry Experiments (M ISFISH IE). O M ICS 10, 205-208 (2006).

10. Spidlen J, Gentleman RC, $\mathrm{H}$ aaland PD et al.: $D$ ata standards for flow cytometry. OMICS 10, 209-214 (2006).

11. Leebens-M ack J, Vision T, Brenner $E$, et al.: Taking the first steps towards a standard for reporting on phylogenies: $M$ inimum Information About a Phylogenetic Analysis (M IAPA). O M ICS 10, 231-237 (2006).

12. Andrews PW, Benvenisty $\mathrm{N}, \mathrm{M}$ CK ay $\mathrm{R}$ et al.: The International Stem C ell Initiative: toward benchmarks for human embryonic stem cell research. $\mathrm{N}$ at. Biotechnol. 23, 795-797 (2005).

13. Strelchenko $N$, Verlinsky 0, Kukharenko V, Verlinsky Y: M orula-derived human embryonic stem cells. Reprod. Biomed. O nline 9, 623-629 (2004).

14. Zhang $X$ Stojkovic $P$, Przyborski $S$ et al.: $D$ erivation of human embryonic stem cells from developing and arrested embryos. Stem Cells (2006) (Epub ahead of print).
15. Klimanskaya I, C hung Y, Becker S, Lu SJ, Lanza R: H uman embryonic stem cell lines derived from single blastomeres. N ature D 0 I 10.1038/nature05142 (2006) (Epub ahead of print).

16. Lanzendorf SE, Boyd CA, Wright DL, $M$ uasher $S$, $O$ ehninger $S$, $H$ odgen $G D$ : U se of human gametes obtained from anonymous donors for the production of human embryonic stem cell lines. Fertil. Steril. 76, 132-137 (2001).

17. H eins N, Englund M C, Sjoblom C et al.: $D$ erivation, characterization, and differentiation of human embryonic stem cells. Stem Cells 22, 367- 376 (2004).

18. Pickering $S$ J, Braude PR, Patel $M$ et al.: Preimplantation genetic diagnosis as a novel source of embryos for stem cell research. Reprod. Biomed. O nline 7, 353-364 (2003).

19. Chen $\mathrm{H}, \mathrm{Q}$ ian $\mathrm{K}, \mathrm{HuJ}$ et al.: The derivation of two additional human embryonic stem cell lines from day 3 embryos with low morphological scores. H um. Reprod. 20, 2201-2206 (2005).

20. M italipova M , Calhoun J, Shin S et al.: $\mathrm{H}$ uman embryonic stem cell lines derived from discarded embryos. Stem Cells 21, 521-526 (2003).

21. Amit M, Itskovitz-Eldor J : D erivation and spontaneous differentiation of human embryonic stem cells. J. Anat. 200, 225-232 (2002).

22. Findikli N, Kahraman S, Akcin O, Sertyel S, C andan Z: Establishment and characterization of new human embryonic stem cell lines. Reprod. Biomed. O nline 10, 617-627 (2005).

23. Inzunza J, G ertow K, Stromberg M A et al.: D erivation of human embryonic stem cell lines in serum replacement medium using postnatal human fibroblasts as feeder cells. Stem Cells 23 , 544-549 (2005).

24. Gardner D K, Lane M, Stevens ], Schlenker T, Schoolcraft W B: Blastocyst score affects implantation and pregnancy outcome: towards a single blastocyst transfer. Fertil. Steril. 73, 1155-1158 (2000).

25. Veeck $L L, Z$ aninovic $N$ : An atlas of human blastocysts. The Parthenon Publishing Group, Lancashire, UK (2003).

26. Richter $\mathrm{KS}, \mathrm{H}$ arris $\mathrm{DC}, \mathrm{D}$ aneshmand $\mathrm{ST}$, Shapiro BS: Q uantitative grading of a human blastocyst: optimal inner cell mass size and shape. Fertil. Steril. 76, 1157-1167 (2001).
27. Arce JC, Ziebe S, Lundin K, Janssens R, $H$ elmgaard $L$, Sorensen $P$ : Interobserver agreement and intraobserver reproducibility of embryo quality assessments. H um. Reprod. 21, 2141-2148 (2006).

28. Gibbons J, H ewitt E, Gardner D K : Effects of oxygen tension on the establishment and lactate dehydrogenase activity of murine embryonic stem cells. Cloning Stem Cells 8, 117-122 (2006).

29. Stojkovic M, Lako M, Strachan T, M urdoch A. D erivation, growth and applications of human embryonic stem cells. Reproduction 128, 259-267 (2004).

30. Fong $C Y$, Richards $M$, Bongso $A$. Unsuccessful derivation of human embryonic stem cell lines from pairs of human blastomeres. Reprod. Biomed. O nline 13, 295-300 (2006).

31. M ason $C, H$ oare $M$ : Regenerative medicine bioprocessing: the need to learn from the experience of other fields. Regenerative M ed. 1, 615-623 (2006).

32. $\mathrm{H}$ arris $\mathrm{N}, \mathrm{M}$ unro $\mathrm{C}$, Patel $\mathrm{B}: \mathrm{N}$ ew guidance on codes of practice, standardized methods and regulations for cell-based therapeutics. Regenerative $M$ ed. 1, 705-707 (2006).

33. Thomson JA, Itskovitz-Eldor J, Shapiro SS et al.: Embryonic stem cell lines derived from human blastocysts. Science 282, 1145-1147 (1998).

34. Reubinoff $B E$, Pera $M F$, Fong $C Y$, Trounson A, Bongso A: Embryonic stem cell lines from human blastocysts: somatic differentiation in vitro. $\mathrm{N}$ at. Biotechnol. 18, 399-404 (2000).

35. Park JH, Kim SJ, O h EJ et al.: Establishment and maintenance of human embryonic stem cells on ST O, a permanently growing cell line. Biol. Reprod. 69, 2007-2014 (2003).

36. H ovatta $\mathrm{O}, \mathrm{M}$ ikkola M, G ertow K et al.: A culture system using human foreskin fibroblasts as feeder cells allows production of human embryonic stem cells. H um. Reprod. 18, 1404-1409 (2003).

37. Kim SJ, Lee JE, Park JH et al.: Efficient derivation of new human embryonic stem cell lines. M ol. Cells 19, 46-53 (2005).

38. Stojkovic M, Lako M, Stojkovic P et al.: $D$ erivation of human embryonic stem cells from day- 8 blastocysts recovered after three-step in vitro culture. Stem Cells 22 , 790-797 (2004).

39. Cowan CA, Klimanskaya I, M CM ahon J et al.: D erivation of embryonic stem-cell lines from human blastocysts. N. Engl J. M ed. 350, 1353-1356 (2004). 
40. Pickering $S$ J, $M$ inger $S L$, Patel $M$ et al.: $G$ eneration of a human embryonic stem cell line encoding the cystic fibrosis mutation $\delta$ F 508, using preimplantation genetic diagnosis. Reprod. Biomed. O nline 10, 390-397 (2005).

41. Kim H S, O h SK, Park YB et al.: M ethods for derivation of human embryonic stem cells. Stem Cells 23, 1228-1233 (2005).

42. Lysdahl $H, G$ abrielsen $A, M$ inger $S L$ et al.: D erivation and characterization of four new human embryonic stem cell lines: the D anish experience. Reprod. Biomed. Online 12, 119-126 (2006).
43. Ludwig TE, Levenstein M E, JonesJM et al.: D erivation of human embryonic stem cells in defined conditions. $\mathrm{N}$ at. Biotechnol. 24, 185-187 (2006).

\section{Websites}

101. www.bsi-

global.com/emergingtechnologies/Regenme d/Benefits.xalter
102. US Pharmacopeia www.usp.org

103. UK Stem Cell Bank www.ukstemcellbank.org.uk

104. The latest news and information on stem cell research www.stemcell.net 